\title{
Combined Therapy Sensitivity Index Based on a 13-Gene Signature Predicts Prognosis for IDH Wild-type and MGMT Promoter Unmethylated Glioblastoma Patients
}

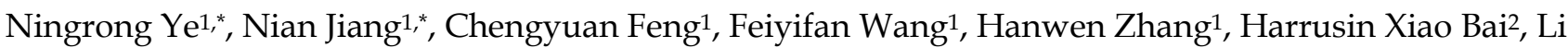 \\ Yang ${ }^{3}$, Yandong Su${ }^{1}$, Chunhai Huang, ${ }^{4}$, Siyi Wanggou ${ }^{1 凶}$, Xuejun $\mathrm{Li}^{1 凶}$ \\ 1. Department of Neurosurgery, Xiangya Hospital, Central South University, Changsha, Hunan, China; \\ 2. Department of Radiology, Hospital of the University of Pennsylvania, Philadelphia, Pennsylvania; \\ 3. Department of Neurology, The Second Xiangya Hospital, Central South University, Changsha, Hunan, China. \\ 4. Department of Neurosurgery, The First Affiliated Hospital of Jishou University, Jishou, Hunan China. \\ * Ningrong Ye and Nian Jiang have contributed equally to this work. \\ $\triangle$ Corresponding author: Xuejun Li M.D., Ph.D., Professor of Neurosurgery, Department of Neurosurgery, Xiangya Hospital, Central South University, 87 \\ Xiangya Road, Changsha, Hunan, 410008, China. Phone: 86731 89753035; Email: 1xjneuro@csu.edu.cn. Siyi Wanggou M.D., Ph.D., Department of Neurosurgery, \\ Xiangya Hospital, Central South University, 87 Xiangya Road, Changsha, Hunan, 410008, China. Email: zokygwong@gmail.com
}

() The author(s). This is an open access article distributed under the terms of the Creative Commons Attribution License (https://creativecommons.org/licenses/by/4.0/). See http:/ /ivyspring.com/terms for full terms and conditions.

Received: 2018.10.12; Accepted: 2019.06.25; Published: 2019.08 .29

\begin{abstract}
Glioblastoma (GBM) is one of the lethal tumors with poor prognosis. However, prognostic prediction approaches need to be further explored. Therefore, we developed an evaluation system that could be used for prognostic prediction of GBM patients. Published mRNA expression datasets from The Cancer Genome Atlas (TCGA), Gene Expression Omnibus (GEO) and Chinese Glioma Genome Atlas (CGGA) were analyzed. Quantitative Realtime-PCR of signature genes and molecular aberrations of 178 Xiangya GBM patients were used for confirmation. Gene set enrichment analysis (GSEA) was performed for functional annotation. As a result, we established a 13-gene signature which named Combined Therapy Sensitivity Index (CTSI). Based on a cutoff point, we divided patients into high-risk group and low-risk group. Based on Kaplan-Meier analysis and multivariate Cox regression analysis, we found that patients in the high-risk group had a shorter overall survival time than patients in the low-risk group ( $p<0.001$ in TCGA and CGGA datasets, $p=0.047$ in GSE4271 dataset, $p=0.008$ in Xiangya GBM cohort, HR: 1.65-3.42). By comparing the status of IDH mutation, TERT promoter mutation (TERTp-mut) and MGMT promoter methylation, CTSI was predictable in IDH wild-type (IDH-wt)/MGMT promoter unmethylated (MGMTp-unmeth) patients $\quad(p=0.037$ in IDH-wt/TERTp-mut/MGMTp-unmeth subgroup, HR: 1.98; $p=0.032$ in IDH-wt/TERTp-wt/MGMTp-unmeth subgroup, HR: 2.09). Based on GESA, the Gene Ontology (GO) gene sets were enriched differently between CTSI high-risk and low-risk groups. Our results showed CTSI risk score can predict the prognosis of IDH-wt/MGMTp-unmeth GBM patients. Based on CTSI, combined with the status of IDH mutation, TERT promoter mutation and MGMT promoter methylation, a stepwise prognosis evaluation system which can provide precise prognosis prediction for GBM patients was established.
\end{abstract}

Key words: Glioblastoma, Gene signature, CTSI, Prognosis

\section{Introduction}

Glioblastoma (GBM) is one of the most common and lethal malignant primary brain tumors in adults with a median survival time of only 14 months. Less than $5 \%$ of the patients live more than 5 years after diagnosis $[1,2]$. The mainstay of treatment is maximal resection, followed by radiation and chemotherapy. Despite multimodality therapy, the outcomes are dismal $[3,4]$. Therefore, novel diagnostic/prognostic biomarkers and better therapeutic targets are urgently needed [5]. 
In the past two decades, IDH mutation, MGMT promoter methylation, TERT promoter mutation, RTK, PI3K and p53 pathways alterations, molecular subtype and G-CIMP phenotype were identified to play crucial roles in the pathogenesis of GBM and also be of prognostic value [6-17]. These studies above made a better understanding of GBM's landscape and disrupted signaling pathways, and provided more practical pathological classifications and personalized treatment regimens [18-22]. However, they can't fully cover all cases such as IDH-wt/MGMTp-unmeth patients. Therefore, unearthing novel prognostic biomarkers, molecular targets and molecular signatures, and illustrating their expression with molecular features of GBM, are meaningful for improving the outcome of GBM patients.

In this study, we analyzed gene expression profiles of GBM patients in TCGA dataset, CGGA dataset and GEO dataset, respectively. By using these datasets, we identified and validated 13 signature genes that have prognostic value and proposed a risk score index named as Combined Therapy Sensitivity Index, CTSI. We tested 178 GBM samples from Xiangya GBM cohort and identified the prognostic value of CTSI in IDH-wt/MGMTp-unmeth patients. As a result, we established a stepwise algorithm for outcome prediction of the all GBM patients from the worst to the best outcome.

\section{Results}

\section{Identification of signature genes for CTSI risk scoring model}

The workflow of data processing and data filtering is shown in Figure 1. 529 TCGA GBM patients' mRNA expression profile from Affymetrix HG-U133 Plus 2.0 was acquired from published database. First, we picked out 1349 GBM tissue specific genes (profile A) of 529 GBM patients comparing to normal brain tissue. Then we chose 195 patients who receive both radio- and chemotherapy and found out 1399 related gene (profile B). After filtering profile $\mathrm{B}$ in profile $\mathrm{A}$, we discovered 137 GBM specific and radio-chemotherapy sensitivity correlated genes. We evaluated the prognostic impact of those genes in training set using univariate Cox regression analysis. Finally, 13 genes were identified as significantly associated with survival $(P<0.01)$ (Supplementary Table S2). And their expression levels were shown in Figure 2A. All these13 genes were further analyzed using multivariable Cox regression analysis. Finally, we calculated a risk score by integrating the gene expression data and the corresponding coefficients derived from the multivariate Cox regression analysis. This risk scoring model was named as Combined Therapy Sensitivity Index (CTSI). The CTSI calculation formula is described as below:

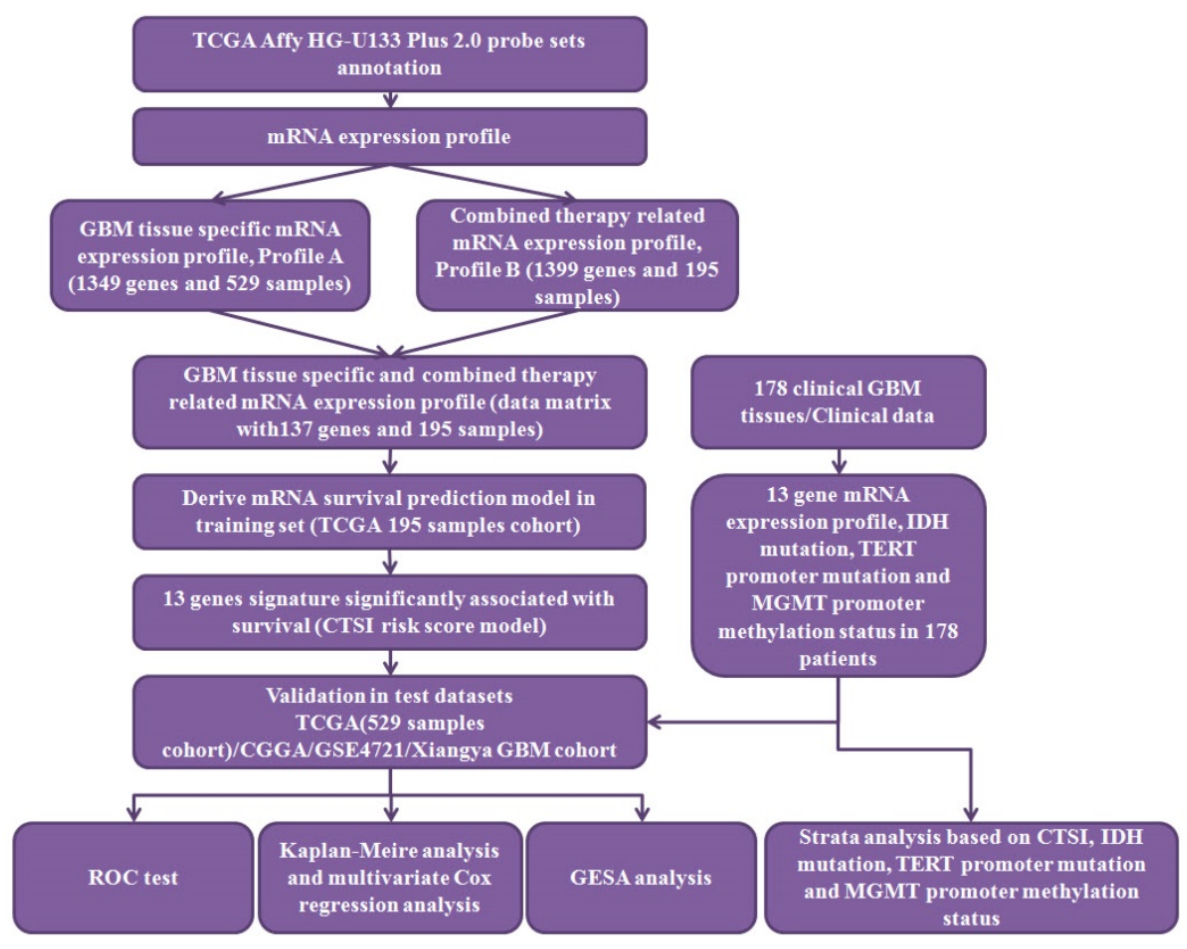

Figure 1. The workflow of data processing. 529 TCGA GBM patients' mRNA expression profile from Affymetrix HG-U133 Plus 2.0 was acquired from published database. By data filtering, a matrix with 137 genes and 195 samples was generated as training set to establish the CTSI risk score model. CGGA GBM dataset, GSE 4721 GBM dataset, TCGA 529 patient cohort and 178 Xiangya GBM cohort were used as validation sets. In Xiangya GBM cohort, TERT promoter mutation, MGMT promoter methylation and IDH mutation were sequenced for strata analysis. 
CTSI risk score $=(-1.18 \times$ PCNA $)+(1.84 \times$ EIF3D $)+$ $(-1.93 \times$ ATP6V1E1 $)+(1.52 \times$ ECHS1 $)+(0.93 \times$ PLOD1 $)+(-0.95 \times$ ERCC1 $)+(0.72 \times$ ADI 1$)+(-0.97 \times$ CALCOCO2 $)+(0.70 \times$ NEDD9 $)+(-1.09 \times \mathrm{MTDH})+$ $(-0.26 \times$ RCAN2 $)+(0.34 \times$ GPNMB $)+(0.87 \times$ VTI1B $)$

We calculated the CTSI risk score for each patient in the training set (TCGA 195 patient cohort) first. Then we ranked them according to their scores and calculated the cutoff point by maximizing Youden index through ROC analysis. As a result, -0.2248 was set as the cutoff point. Subsequently, the patients were divided into CTSI low-risk $(n=91)$ and CTSI high-risk $(n=104)$ groups (Supplementary Figure S1).
A

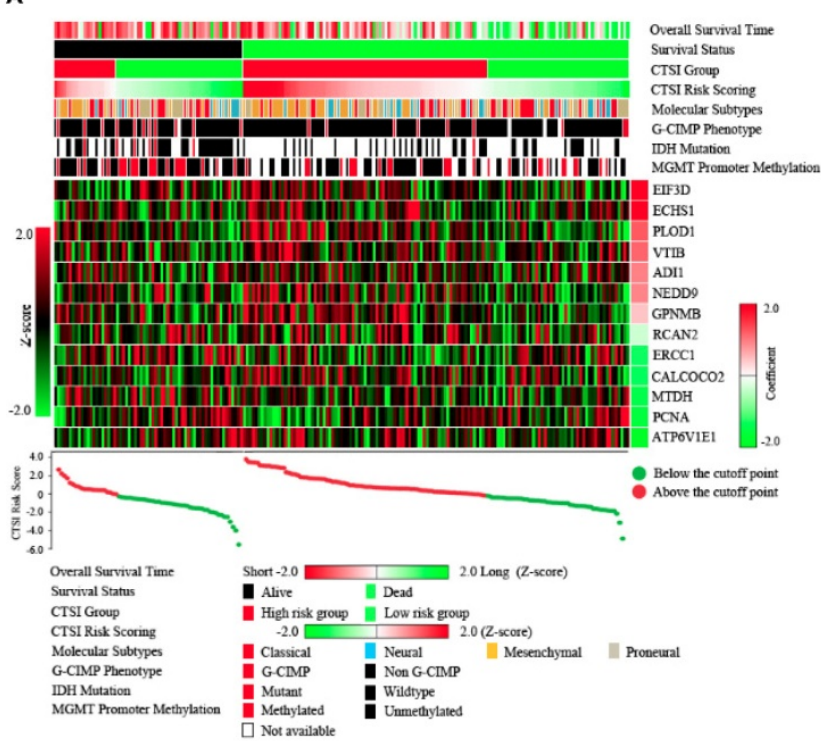

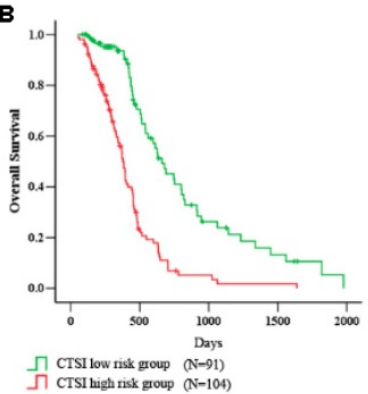

Median OS of patients divided by CTSI risk score

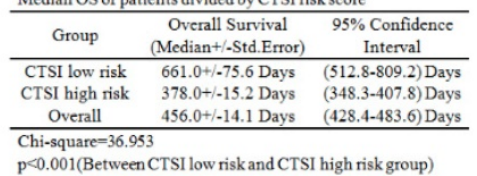

C

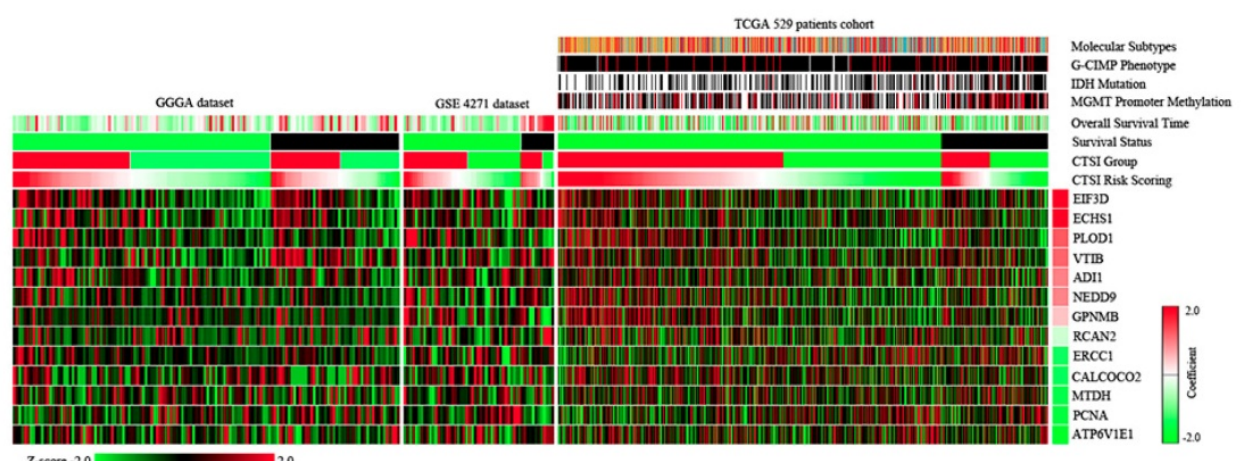

Z-scose-2.0 2.0

Overall Survival Time Short-20 20 Long (Z-scose)

Survival Status

CTS1 Group

CTSI Risk Scoring

Molecular Subtypes

IDH Mutation

1. Alive Dead

1. High nisk group ||- Low nisk group

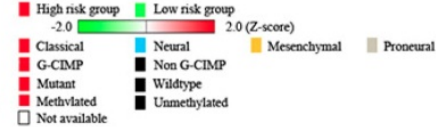

D

E
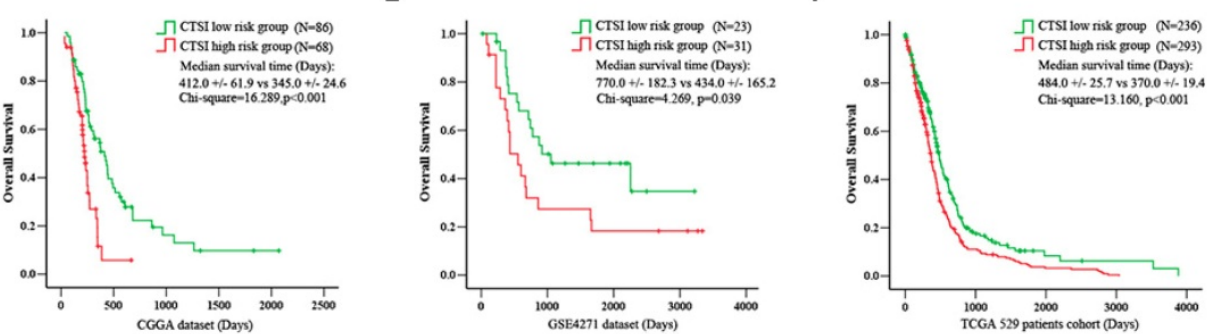

Figure 2. Prognostic value of CTSI risk score. A. Distribution of gene expression, CTSI risk score, overall survival and molecular features in TCGA 195 patient cohort. Overall survival time, CTSI risk scoring and expression of signature genes were converted into Z-score to regain the heatmap. Survival status, CTSI group, molecular subtypes, G-CIMP phenotypes, IDH mutation and MGMT promoter methylation were added for annotation. Signature genes were ranked by coefficients from multivariate Cox regression analysis. The CTSI risk score of each patient was plotted under the heatmap. B. Kaplan-Meier analysis for CTSI risk score model in TCGA 195 patient cohort. C. Distribution of gene expression, CTSI risk score, overall survival and molecular pathological features in CGGA dataset, GSE4271 dataset and TCGA 529 patient cohort. Kaplan-Meier analysis revealed the prognostic value of CTSI risk score model in CGGA dataset (D), GSE4271 dataset (E) and TCGA 529 patient cohort (F). 
A significant difference in overall survival (OS) between the CTSI high-risk group and the low-risk group. The median OS in the high-risk group was 378.0 days versus 661.0 days in the low-risk group $(p<0.001)$ (Figure 2B). We also performed multivariate Cox regression analysis to examine the independence of CTSI risk score on prognosis prediction. Clinical data including age, gender, KPS, extent of resection,
G-CIMP phenotype and molecular subtype were used for multifactor analysis. The result showed that G-CIMP phenotype and CTSI risk score were both prognostic for GBM patients' prognosis. The coefficient of CTSI risk score was 1.26 with the hazard ratio (HR) at 3.54 (95\% confidence interval: 2.26-5.46) (Table 1).

Table 1. Cox proportional hazard model for overall survival

\begin{tabular}{|c|c|c|c|c|c|c|c|c|}
\hline & \multicolumn{4}{|c|}{$\begin{array}{l}\text { Univariate Cox regression } \\
\text { Method: Enter }\end{array}$} & \multicolumn{4}{|c|}{$\begin{array}{l}\text { Multivariate Cox regression } \\
\text { Method: Backward likewise }\end{array}$} \\
\hline & Co.ef & Std.Err & P value & HR $(95 \%$ CI $)$ & Co.ef & Std.Err & $\mathrm{P}$ value & HR $(95 \%$ CI) \\
\hline \multicolumn{9}{|l|}{ TCGA 195 patients cohort } \\
\hline Age & 0.80 & 0.11 & $<0.001$ & $1.33(1.09-1.62)$ & & & & \\
\hline Gender & 0.10 & 0.10 & 0.34 & $2.33(1.79-3.05)$ & & & & \\
\hline KPS & -0.85 & 0.14 & $<0.001^{*}$ & $0.45(0.36-0.56)$ & -0.46 & 0.26 & 0.081 & $0.63(0.37-1.06)$ \\
\hline Surgical Resection & 0.24 & 0.24 & 0.316 & $1.27(0.78-2.02)$ & & & & \\
\hline G-CIMP & -1.15 & 0.20 & $<0.001^{*}$ & $0.81(0.70-0.95)$ & -1.12 & 0.21 & $<0.001^{*}$ & $0.80(0.69-0.96)$ \\
\hline Molecular subtype & 0.01 & 0.10 & 0.892 & $1.01(0.83-1.24)$ & & & & \\
\hline \multirow[t]{2}{*}{ CTSI risk score } & 1.27 & 0.24 & $<0.001^{*}$ & $3.55(2.23-5.64)$ & 1.26 & 0.22 & $<0.001^{*}$ & $3.54(2.26-5.46)$ \\
\hline & \multicolumn{8}{|c|}{ No. of subject $=161$, No. of event $=106$, No. of censored=55, Log likelihood=835.008 } \\
\hline \multicolumn{9}{|c|}{ TCGA 529 patients cohort } \\
\hline Age & 0.53 & 0.14 & $<0.001^{*}$ & $1.69(1.29-2.21)$ & 0.55 & 0.14 & $<0.001^{*}$ & $1.73(1.33-2.26)$ \\
\hline Gender & -0.08 & 0.13 & 0.558 & $0.93(0.72-1.19)$ & & & & \\
\hline KPS & -3.34 & 0.15 & $0.027^{*}$ & $0.71(0.53-0.96)$ & -0.34 & 0.15 & $0.021^{*}$ & $0.71(0.53-0.95)$ \\
\hline Surgical Resection & 0.14 & 0.17 & 0.398 & $1.15(0.83-1.61)$ & & & & \\
\hline G-CIMP & -1.15 & 0.26 & $<0.001^{*}$ & $0.32(0.19-0.53)$ & -0.99 & 0.24 & $<0.001^{*}$ & $0.37(0.23-0.59)$ \\
\hline Molecular subtype & 0.07 & 0.06 & $0.024^{*}$ & $1.07(0.95-1.21)$ & & & & \\
\hline Treatment modality & -3.45 & 0.234 & 0.137 & $0.71(0.45-1.12)$ & -0.39 & 0.23 & 0.084 & $0.67(0.43-1.06)$ \\
\hline \multirow[t]{2}{*}{ CTSI risk score } & 0.49 & 0.13 & $<0.001^{*}$ & $1.63(1.27-2.09)$ & 0.49 & 0.13 & $<0.001^{*}$ & $1.65(1.29-2.11)$ \\
\hline & \multicolumn{8}{|c|}{ No. of subject $=369$, No. of event $=279$, No. of censored $=90$, Log likelihood $=2722.843$} \\
\hline \multicolumn{9}{|l|}{ GSE4271 dataset } \\
\hline Age & 1.77 & 1.58 & 0.263 & $5.89(1.26-9.83)$ & & & & \\
\hline Gender & -0.41 & 0.82 & 0.619 & $0.67(0.13-3.31)$ & & & & \\
\hline Necrosis & -0.09 & 1.19 & 0.939 & $0.91(0.09-9.36)$ & & & & \\
\hline \multirow[t]{2}{*}{ CTSI risk score } & 2.28 & 1.22 & $0.048^{*}$ & $5.73(1.18-9.31)$ & 1.54 & 0.84 & $0.047^{*}$ & $4.65(1.12-8.02)$ \\
\hline & \multicolumn{8}{|c|}{ No. of subject $=54$, No. of event $=42$, No. of censored $=12$, Log likelihood $=424.526$} \\
\hline \multicolumn{9}{|l|}{ CGGA dataset } \\
\hline Recurrence & 0.41 & 0.22 & 0.059 & $1.50(0.98-2.29)$ & & & & \\
\hline \multirow[t]{2}{*}{ CTSI risk score } & 0.99 & 0.24 & $<0.001^{*}$ & $2.69(1.68-4.32)$ & 0.94 & 0.34 & $<0.001^{*}$ & $2.56(1.56-4.08)$ \\
\hline & \multicolumn{8}{|c|}{ No. of subject $=138$, No. of event $=92$, No. of censored $=46$, Log likelihood $=743.422$} \\
\hline \multicolumn{9}{|l|}{ Xiangya GBM cohort } \\
\hline Age & 0.48 & 0.19 & $0.016^{*}$ & $1.61(1.09-2.38)$ & 0.48 & 0.19 & $0.016^{*}$ & $1.61(1.09-2.38)$ \\
\hline Gender & -0.55 & 0.45 & 0.221 & $0.58(0.24-1.39)$ & & & & \\
\hline KPS & -0.48 & 0.21 & $0.021^{*}$ & $0.62(0.41-0.93)$ & -0.48 & 0.21 & $0.021^{*}$ & $0.62(0.41-0.93)$ \\
\hline Surgical Resection & 0.01 & 0.32 & 0.970 & $1.01(0.54-1.88)$ & & & & \\
\hline Treatment modality & -0.82 & 0.22 & $<0.001^{*}$ & $0.44(0.29-0.68)$ & -0.82 & 0.22 & $<0.001^{*}$ & $0.44(0.29-0.67)$ \\
\hline CTSI risk score & 0.52 & 0.19 & $0.008^{*}$ & $1.68(1.14-2.46)$ & 0.52 & 0.19 & $0.008^{*}$ & $1.68(1.15-2.46)$ \\
\hline $\begin{array}{l}\text { TERT promoter } \\
\text { mutation }\end{array}$ & 0.62 & 0.20 & $0.003^{*}$ & $1.85(1.24-2.76)$ & 0.61 & 0.20 & $0.003^{*}$ & $1.85(1.24-2.75)$ \\
\hline $\begin{array}{l}\text { MGMT promoter } \\
\text { methylation }\end{array}$ & -0.98 & 0.24 & $<0.001^{*}$ & $0.38(0.23-0.61)$ & -0.98 & 0.24 & $<0.001^{*}$ & $0.38(0.23-0.61)$ \\
\hline \multirow[t]{2}{*}{ IDH mutation } & -1.98 & 0.62 & $0.002^{*}$ & $0.14(0.04-0.47)$ & -1.97 & 0.62 & $0.001^{*}$ & $0.14(0.04-0.47)$ \\
\hline & \multicolumn{8}{|c|}{ No. of subject $=178$, No. of event $=122$, No. of censored $=56$, Log likelihood $=1014.674$} \\
\hline
\end{tabular}

Age: $0=$ Age $<65$ years, $1=$ Age $>65$ years .

Gender: $0=$ Female, $1=$ Male.

KPS: $0=$ KPS $<70,1=$ KPS $>=70$.

Surgical resection: $0=$ Others, $1=$ total resection.

G-CIMP: $0=$ Non-C-CIMP, $1=$ G-CIMP

Molecular subtype: $0=$ Non-proneural subtype. $1=$ Proneural subtype.

Treatment modality: $0=$ Without chemotherapy or radiotherapy, $1=$ Chemotherapy with/or radiotherapy.

Necrosis: $0=$ No necrosis, $1=$ Necrosis .

Recurrence: $0=$ No recurrence, $1=$ Recurrence.

CTSI risk score: $0=$ Low risk score group; $1=$ High risk score group.

TERT promoter mutation: $0=$ Wildtype; $1=$ Mutated.

MGMT promoter methylation: $0=$ Unmethylated; $1=$ Methylated

IDH mutation: $0=$ Wildtype; $1=$ Mutated.

${ }^{*}, \mathrm{p}<0.05$ accepted as significance. 


\section{Validation of CTSI risk score on survival prediction}

To examine the reliability of CTSI on survival prediction, we analyzed the CGGA dataset $(n=144)$, the GSE4271 dataset $(n=54)$ and all GBM patients in the TCGA dataset $(n=529)$ as validation dataset. Based on the CTSI cutoff point defined by the training dataset, patients in all these validation datasets were divided into CTSI high-risk group and CTSI low-risk group, respectively. The Kaplan-Meier survival analysis verified that patients in the low-risk group had better prognosis in each dataset (median OS, days, CTSI low-risk vs CTSI high-risk: $412.0+$ /-61.9 vs $345.0+/-24.6, \quad \mathrm{p}<0.001, \quad$ in CGGA dataset; $770.0+/-182.3$ vs $434.0+/-165.2, p=0.039$, in GES4271 dataset; $484.0+/-25.7$ vs $370.0+/-19.4, p<0.001$, in TCGA 529 patients cohort) (Figure 2 C, D, E \& F). More importantly, the multivariate Cox regression analysis confirmed that the CTSI risk score was a significant predictive factor. The hazard ratio of CTSI high-risk group ranged from 1.45 to 4.65 (Table 1).

\section{Evaluation of CTSI risk score performance}

We performed receiver operating characteristic (ROC) analysis to examine the sensitivity and specificity of CTSI on prognosis prediction, using MGMT promoter methylation, IDH mutation and TERT promoter mutation as references. The area under the curve (AUC) of CTSI was $0.820(p=0.004)$ (Supplementary Figure S2A).

\section{IDH mutation, MGMT promoter methylation, TERT promoter mutation and CTSI risk score}

To further investigate the influence of MGMT promoter methylation, IDH mutation and TERT promoter mutation on prognostic value of CTSI, we performed subgroup analysis based on TCGA dataset. For IDH-wt patients, the high CTSI risk score was associated with the worse outcome (median OS, days, CTSI low-risk vs CTSI high-risk: $422.0+/-24.1$ vs $357.0+/-20.3, p=0.012$ ). However, for IDH-mut patients, the CTSI risk score cannot show prognostic value (Supplementary Figure S2B). For MGMTp-unmeth patients, CTSI low-risk score group showed a better prognosis (median OS, days, CTSI low-risk vs CTSI high-risk:485.0+/-34.7 days vs $362.0+/-23.0, p=0.013)$. However, for MGMTp-meth patients, there was no significant difference (Supplementary Figure S2C). For TERT promoter mutation, patients in the CTSI high-risk group had shorter overall survival time in both strata (median OS, days, CTSI low-risk vs CTSI high-risk: $484.0+/-45.2$ vs $382.0+/-34.8, p=0.018$, in TERTp-wt group; $485.0+/-46.7$ vs $372.0+/-24.2, \mathrm{p}=0.002$, in TERTp-mut group) (Supplementary Figure S2D).
These results demonstrated that CTSI risk score can predict the prognosis of IDH-wt/MGMTp-unmeth GBM patients.

\section{Combined CTSI risk score and TERT promoter mutation for prognosis prediction in IDH-wt and MGMTp-unmeth patients}

For further validation, we performed quantitative real-time PCR for the 13 signature genes and detected the status of IDH mutation, TERT promoter mutation and MGMT promoter methylation in 178 Xiangya GBM patients. Compared with normal brain tissue, PCNA, CALCOCO2, ADI1, GPNMB, $\mathrm{MTDH}$, EIF3D, NEDD9, ERCC1 and PLOD1 were up-regulated while ATP6V1E1, VTI1B, ECHS1 and RCAN2 were down-regulated in GBM patients (Supplementary Figure S3). The mutation rate of IDH was only $6.2 \%$. Most of the GBM patients showed an IDH-wt phenotype. The TERT promoter had a mutation rate of $46.6 \%$. The methylation rate of MGMT promoter was $25.8 \%$ (Supplementary Table S3). The mRNA expression of these 13 genes are transferred into $\mathrm{z}$ score and plotted in heatmap as shown in Figure 3A.

We validated the prognostic value of CTSI risk score in all patients of Xiangya GBM cohort. The median OS of CTSI high-risk group was 350.0+/-22.6 days versus $455.0+/-22.6$ days in CTSI low-risk group $(\mathrm{p}=0.002)$ (Figure $3 \mathrm{~B})$. CTSI was a significant prognostic factor on multivariate Cox regression analysis with a hazard ratio of 1.68 for CTSI high-risk group (Table 1).

For IDH-wt patients, depending on TERT promoter mutation and MGMT promoter methylation together, we divided these patients into four subgroups: TERT promoter mutated (TERTp-mut)/ MGMTp-unmeth, TERT promoter wild-type (TERTpwt)/MGMTp-unmeth, TERTp-mut/MGMTp-meth and TERTp-wt/MGMTp-meth. The Kaplan-Meier analysis showed that the pronosis of TERTp-mut/ MGMTp-unmeth subgroup was the worst, the TERTp-wt/MGMTp-unmeth subgroup was moderate while other two subgroups were relatively good (Figure 3C). Pairwise comparison between each subgroup showed that for MGMTp-meth patients, the status of TERT mutation didn't affect the OS (Figure 3C). These results were similar as reported by Hideyuki Arita et al. previously [23].

Next, we tested the prognostic value of CTSI in each of the four subgroups. We found that in both TERTp-mut/MGMTp-unmeth and TERTp-wt/ MGMTp-unmeth subgroups, CTSI was an independent prognostic factor on Kaplan-Meier analysis (median OS, CTSI low-risk vs CTSI high-risk: $294.0+/-42.2$ vs $185.0+/-32.1$ days, $\mathrm{p}=0.045$, in 
TERTp-mut/MGMTp-unmeth subgroup; 485.0+ / -22.5 vs $314.0+/-42.8$ days, $p=0.012$, in TERTp-wt/MGMTp-unmeth subgroup) and multivariate Cox regression analysis (CTSI low-risk vs CTSI high-risk: $p=0.037, \mathrm{HR}=1.98$, in TERTp-mut/ MGMTp-unmeth subgroup; $p=0.032, H R=2.09$, in TERTp-wt/MGMTp-unmeth subgroup) (Figure 3D,E, Table 2 and Table 3). For TERTp-mut/MGMTp-meth and TERT-wt/MGMTp-unmeth subgroups, CTSI risk score was not a significant prognostic factor (Figure 3F and Figure 3G).

Then we combined CTSI and TERT promoter mutation in IDH-wt/MGMTp-unmeth patients and divided these patients into four subgroups: TERTp-mut/CTSI-low, TERTp-mut /CTSI-high, TERTp-wt/CTSI-low and TERTp-wt/CTSI-high. The TERTp-wt/CTSI-low subgroup had the best outcome, while the TERTp-mut/CTSI-high subgroup suffered the worst outcome (Figure $3 \mathrm{H}$ ).
A.

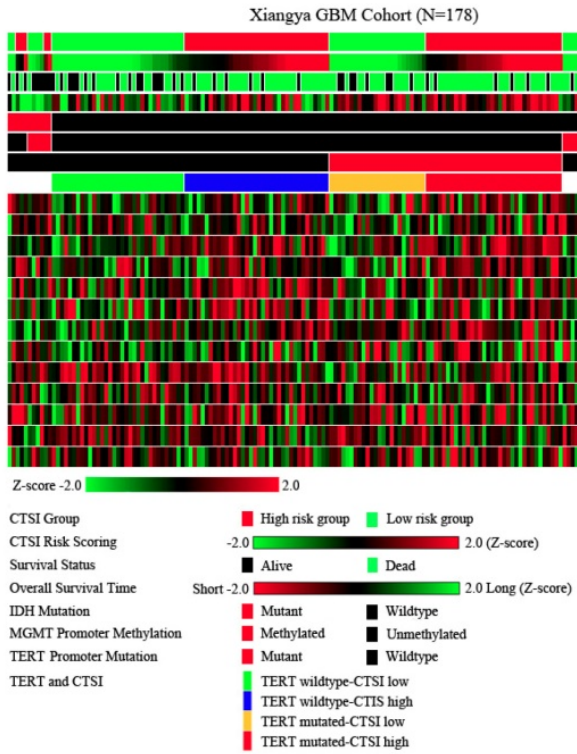

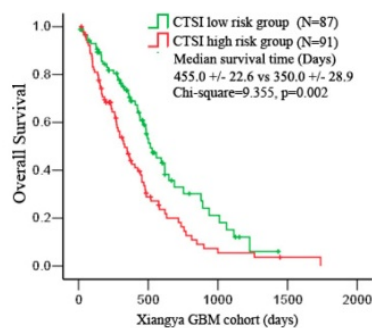

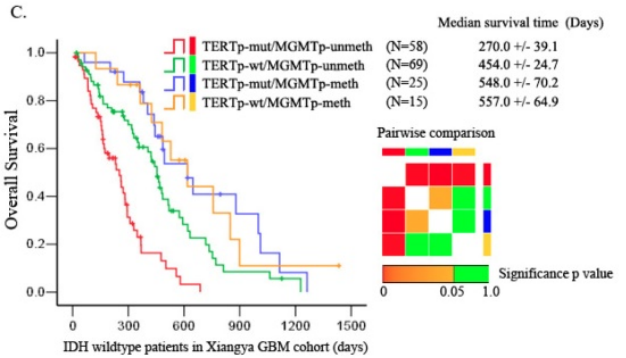

D.
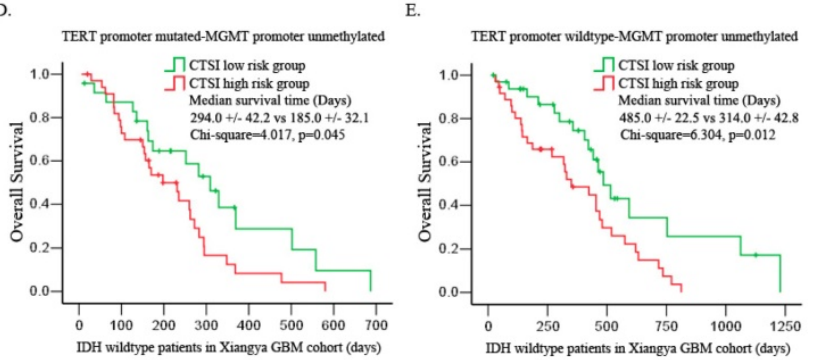

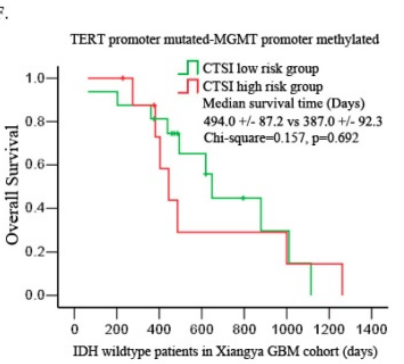

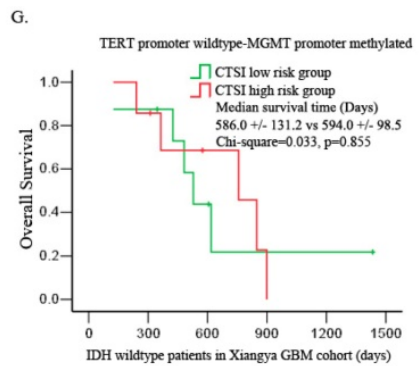

H.

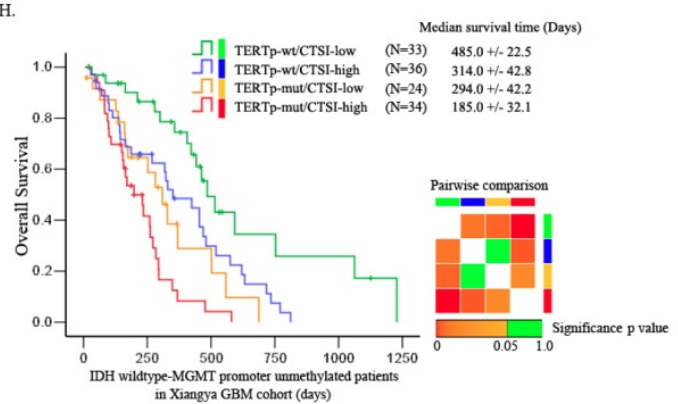

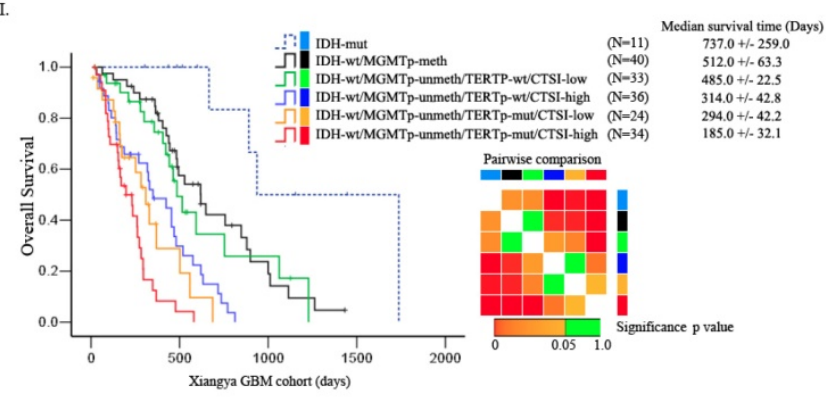

Figure 3. Identification of prognostic value of CTSI risk score in IDH-wt/MGMTp-unmeth patients. A. Distribution of gene expression, CTSI risk score, overall survival, IDH mutation, MGMT promoter methylation and TERT promoter mutation in Xiangya GBM cohort. B. Overall survival of the two groups according to CTSI risk score. C. Pairwise comparison of subgroups' overall survival according to TERT promoter mutation and MGMT promoter methylation for IDH-wt GBM patients. TERT promoter mutation was a prognostic factor for MGMTp-unmeth patients, but not for MGMTp-meth patients. D. Overall survival of CTSI in IDH-wt/TERTp-mut/MGMTp-unmeth patients. E. Overall survival of CTSI in IDH-wt/TERTp-wt/MGMTp-unmeth patients. F. Overall survival of CTSI in IDH-wt/TERTp-mut/MGMTp-meth patients. G. Overall survival of CTSI in IDH-wt/TERTp-wt/MGMTp-meth patients. H. Pairwise comparison of subgroups' overall survival based on TERT promoter mutation and CTSI in IDH-wt/MGMTp-unmeth patients. CTSI was a distinct prognostic factor in both the TERTp-wt and TERTp-mut subgroups. I. Survival of the six subgroups combining IDH mutation, TERT promoter mutation, MGMT promoter methylation and CTSI risk score together. IDH-mut patients received the best outcomes and IDH-wt/MGMTp-unmeth/TERTp-mut/CTSI-high patients received the worst. 
Table 2. Cox proportional hazard model for IDH wildtype, TERT promoter mutated and MGMT promoter unmethylated patients $(\mathrm{No}$. of subject $=58$, No. of event $=44$, No. of censored=14, Log likelihood=269.149).

\begin{tabular}{|c|c|c|c|c|c|c|c|c|}
\hline & \multicolumn{4}{|c|}{$\begin{array}{l}\text { Univariate Cox regression } \\
\text { Method: Enter }\end{array}$} & \multicolumn{4}{|c|}{$\begin{array}{l}\text { Multivariate Cox regression } \\
\text { Method: Backward likewise }\end{array}$} \\
\hline & Co.ef & Std.Err & $\begin{array}{l}\mathrm{P} \\
\text { value }\end{array}$ & $\begin{array}{l}\mathrm{HR}(95 \% \\
\mathrm{CI})\end{array}$ & Co.ef & Std.Err & $\begin{array}{l}\mathrm{P} \\
\text { value }\end{array}$ & $\begin{array}{l}\mathrm{HR}(95 \% \\
\mathrm{CI})\end{array}$ \\
\hline Age & 0.05 & 0.37 & 0.895 & $\begin{array}{l}1.05 \\
(0.51-2.18)\end{array}$ & & & & \\
\hline Gender & 0.28 & 0.35 & 0.415 & $\begin{array}{l}1.33 \\
(0.67-2.66)\end{array}$ & & & & \\
\hline KPS & -0.01 & 0.33 & 0.969 & $\begin{array}{l}0.98 \\
(0.51-1.92)\end{array}$ & & & & \\
\hline $\begin{array}{l}\text { Surgical } \\
\text { Resection }\end{array}$ & -0.44 & 0.58 & 0.450 & $\begin{array}{l}0.65 \\
(0.21-1.99)\end{array}$ & & & & \\
\hline $\begin{array}{l}\text { Treatment } \\
\text { modality }\end{array}$ & -1.19 & 0.38 & $0.002^{*}$ & $\begin{array}{l}0.30 \\
(0.14-0.65)\end{array}$ & -1.06 & 0.33 & $0.001^{*}$ & $\begin{array}{l}0.35 \\
(0.18-0.67)\end{array}$ \\
\hline $\begin{array}{l}\text { CTSI risk } \\
\text { score }\end{array}$ & 0.63 & 0.37 & 0.091 & $\begin{array}{l}1.87 \\
(0.90-3.86)\end{array}$ & 0.68 & 0.33 & $0.037^{*}$ & $\begin{array}{l}1.98 \\
(1.04-3.75)\end{array}$ \\
\hline \multicolumn{9}{|c|}{ Age: $0=$ Age $<65$ years, $1=$ Age $>65$ years . } \\
\hline \multicolumn{9}{|c|}{ Gender: $0=$ Male; $1=$ Female. } \\
\hline \multicolumn{9}{|c|}{ KPS: $0=$ KPS $<70,1=$ KPS $>=70}$. \\
\hline \multicolumn{9}{|c|}{ Surgical resection: $0=$ Others, $1=$ total resection. } \\
\hline \multicolumn{9}{|c|}{$\begin{array}{l}\text { Treatment modality: } 0=\text { Without chemotherapy or radiotherapy, } 1=\text { Chemotherapy } \\
\text { with radiotherapy. }\end{array}$} \\
\hline \multicolumn{9}{|c|}{ CTSI risk score: $0=$ low risk score group $; 1=$ high risk score group. } \\
\hline
\end{tabular}

Table 3. Cox proportional hazard model for IDH wildtype, TERT promoter wildtype and MGMT promoter unmethylated patients (No. of subject $=67$, No. of event $=46$, No. of censored=21, Log likelihood=293.099).

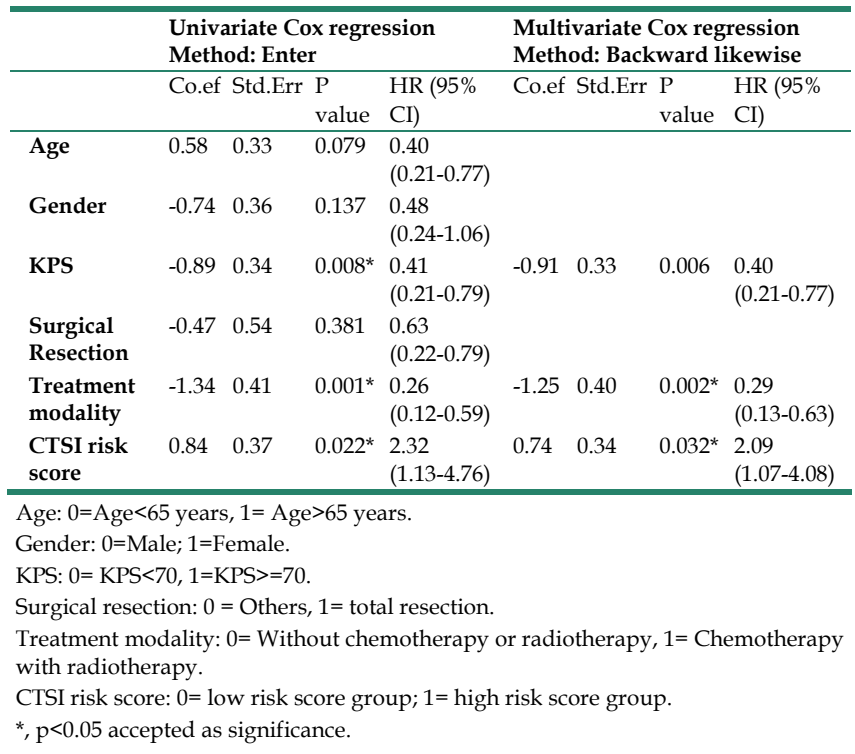

Finally, combing IDH mutation, MGMT promoter methylation, TERT promoter mutation and CTSI risk score together, we made pairwise comparisons among these subgroups. We found that the IDH-mut patients had the best outcome followed by IDH-wt/MGMTp-meth patients, IDH-wt/ MGMTp-meth patients, IDH-wt/MGMTp-unmeth/ TERTp-wt/CTSI-low patients, IDH-wt/MGMTpunmeth/TERTp-wt/CTSI-high patients and IDH-wt/ MGMTp-unmeth/TERTp-mut/CTSI-low patients.
IDH-wt/MGMTp-unmeth/TERTp-mut/CTSI-high patients had the worst outcome (Figure 3I).

\section{Functional annotation of CTSI phenotypes}

For Gene Set Enrichment Analysis, tumors were classified into CTSI high-risk and CTSI low-risk phenotypes. According to GO gene sets, 1842 out of 4058 gene sets were enriched in the CTSI high risk phenotype, while others were enriched in CTSI low-risk phenotype. 674 gene sets in the CTSI high-risk phenotype and 369 gene sets in the CTSI low-risk phenotype were significantly enriched $(P<0.05)$. Many differences were unearthed between two phenotypes on Go biological process, GO cellular component and GO molecular function. Detailed information including plot of p-value vs NES, global ES histogram, heat map and gene list correlation profiles were exhibit exhibited in Supplementary Figure S4.

For GO biological process, the top 20 enriched gene sets were shown in Figure 4A. The extracellular structure organization was most enriched process in CTSI high-risk group and neurotransmitter transport was most enriched process in CTSI low-risk group (Figure 4B and Figure 4C). Interestingly, CTSI high-risk phenotype show intense immunomodulatory including regulation of phagocytosis, inflammatory response, regulation of leukocyte migration, toll like receptor signaling pathway, granulocyte migration, regulation of mast cell activation, and so on (Supplementary Table S4). Besides inflammatory, CTSI high-risk phenotype also tend to closely communicate to environment such as extracellular structure organization, cellular response to biotic stimulus, multicellular organismal macromolecule metabolic process. Meanwhile, CTSI low-risk phenotype was found to related to neurobiological activity including neurotransmitter transport, presynaptic process involved in synaptic transmission, and cell proliferation including DNA biosynthetic process, DNA replication initiation, DNA repair, DNA recombination, microtubule based movement, sister chromatid segregation, cell cycle phase transition, and so on. The biological process of CTSI high- risk and low-risk phenotype reveal different function features, that is CTSI high-risk phenotype were prone to react to extracellular environment while low-risk phenotype tended to focus on cell activity especially proliferation. This can also be applied to GO cellular component and GO molecular function. As to component function, CTSI high-risk phenotype also tended to show high enrichment in function related to extracellular support structure, such as extracellular matrix component, basement membrane, external side of 
plasma membrane, protein complex involved in cell adhesion., while CTSI low-risk phenotype was more likely to show to intracellular structures such as condensed chromosome, chromosomal region, microtubule organizing center part. Referring to molecular function, CTSI high- risk phenotype was enriched in extracellular signal delivery such as integrin binding, cytokine receptor activity, growth factor binding, while low-risk phenotype was enriched intracellular activities such as microtubule motor activity, tubulin binding, DNA helicase activity (Supplementary Figure S5).

The enrichment map revealed that CTSI high-risk phenotype were active to environment response which may contribute to immune escape while CTSI low-risk phenotype was more excited in cancer cell proliferation including mitosis, DNA and RNA metabolism, epigenetic modification, and cytoskeleton/microtubule metabolism (Figure 5 and Supplementary Figure S6).

\section{Discussion}

As the most common primary intracranial malignancy, GBM is revealed as a molecularly heterogeneous disease. Recent studies through novel molecular platforms have provided molecular classifications of this tumor. In this study, based on transcriptome profile, we established a risk score model based on a 13-gene signature and validated its prognostic value by using differently published datasets and one retrospective clinical cohort from our local institution. Our findings of CTSI risk score provided a novel approach for prognosis evaluation of GBM patients and can serve as an important addition to the contemporary GBM prognosis evaluation system.

Recently, several gene signatures have been shown to be associated with the prognosis of glioma patients. Zhang et al. discovered that GPR85, SHOX2 and HMBOX1 had both diagnostic and prognostic values for patients with anaplastic glioma [24]. Wang et al. reported a 3-gene signature (FPR3, IKBIP and S100A9) for prognostic evaluation of MGMTp-meth GBM patients [25]. Compared with Wang's study, CTSI also provided good prognostic prediction of IDH-wt/MGMTp-unmeth patients. Consequently, it serves good complement to the 3-gene signature by Wang et al. to portrait the overall prognosis prediction map for GBM patients.

Out of the 13 signature genes, ATP6V1E1, EIF3D, ERCC1, GPNMB, MTDH, PCNA and NEDD9 have been reported to play crucial roles in various pathways and mechanism of glioma. Some of them were associated with prognosis [26-32]. Other genes, such as CALCOCO2, RCAN2, ADI1, ECHS1, PLOD1 and VTI1B were associated with tumorigenesis or angiogenesis in some other cancers [33-35]. Our study is the first one to report the involvement of those genes in GBM patients.

Gene set enrichment analysis revealed important genetic differences between the CTSI high-risk group and the CTSI low-risk group in our study. We found more than 1000 signaling pathways and biological processes that were differentially enriched in the two phenotypes. Gene sets related to cell cycle, mitosis, mRNA regulation, transcription, translation, DNA replication, DNA repair, neural biological processes and neural development were enriched in CTSI low-risk phenotype, while gene sets enriched in CTSI high-risk phenotype were mainly involved in
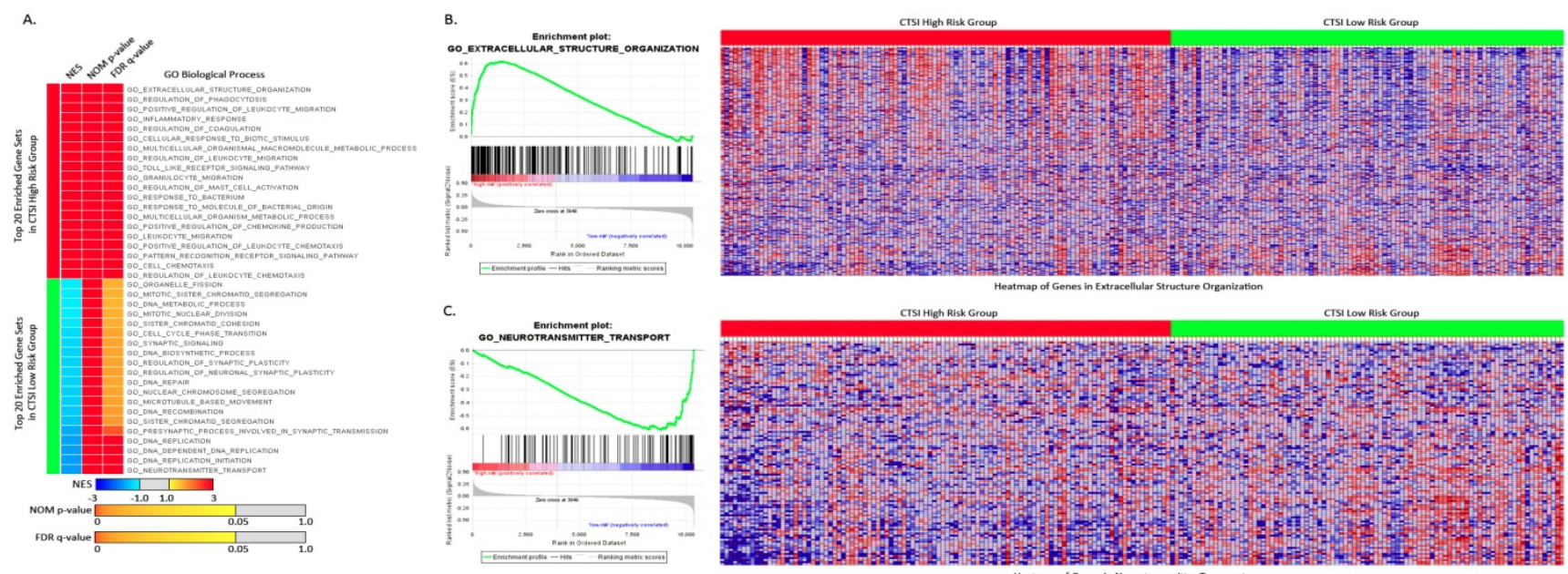

Figure 4. Enriched gene sets of GO biological process based on both CTSI risk score phenotypes. A. Top 20 up-regulated gene sets of GO biological process in both CTSI high-risk phenotype and CTSI low-risk phenotype. EXTRACELLULAR STRUCTURE ORGANIZATION was with the highest normalized enrichment scores (NES) in CTSI high-risk phenotype. NEUROTRANSMITTER TRANSPORT was the most enriched gene set in CTSI low-risk phenotype. Statistics as NES, nominal p-value (NOM p-value) and false discovery rate q-value (FDR q-value) were also shown. B. GSEA enriched profile and heatmap of enriched EXTRACELLULAR STRUCTURE ORGANIZATION in CTSI high-risk score phenotype. C. GSEA enriched profile and heatmap of enriched NEUROTRANSMITTER TRANSPORT in CTSI low-risk score phenotype. 
immune responses, cell surface interactions, extracellular matrix, cell junction, cellular responses, apoptosis and energy metabolism. These genetic differences may offer some insights into the reason why GBM patients can have drastically different prognosis.

IDH mutation, MGMT promoter methylation and TERT promoter mutation are widely recognized as prognostic biomarkers in GBM patients. Patients with IDH mutation or wild-type TERT promoter have better outcomes compared with their counterparts $[9,14]$. Patients with MGMT promoter methylation are more sensitive to temozolomide therapy [36]. According to our analysis, CTSI risk score was unable to predict the prognosis of IDH-mut patients and MGMTp-meth patients. This indicates that the
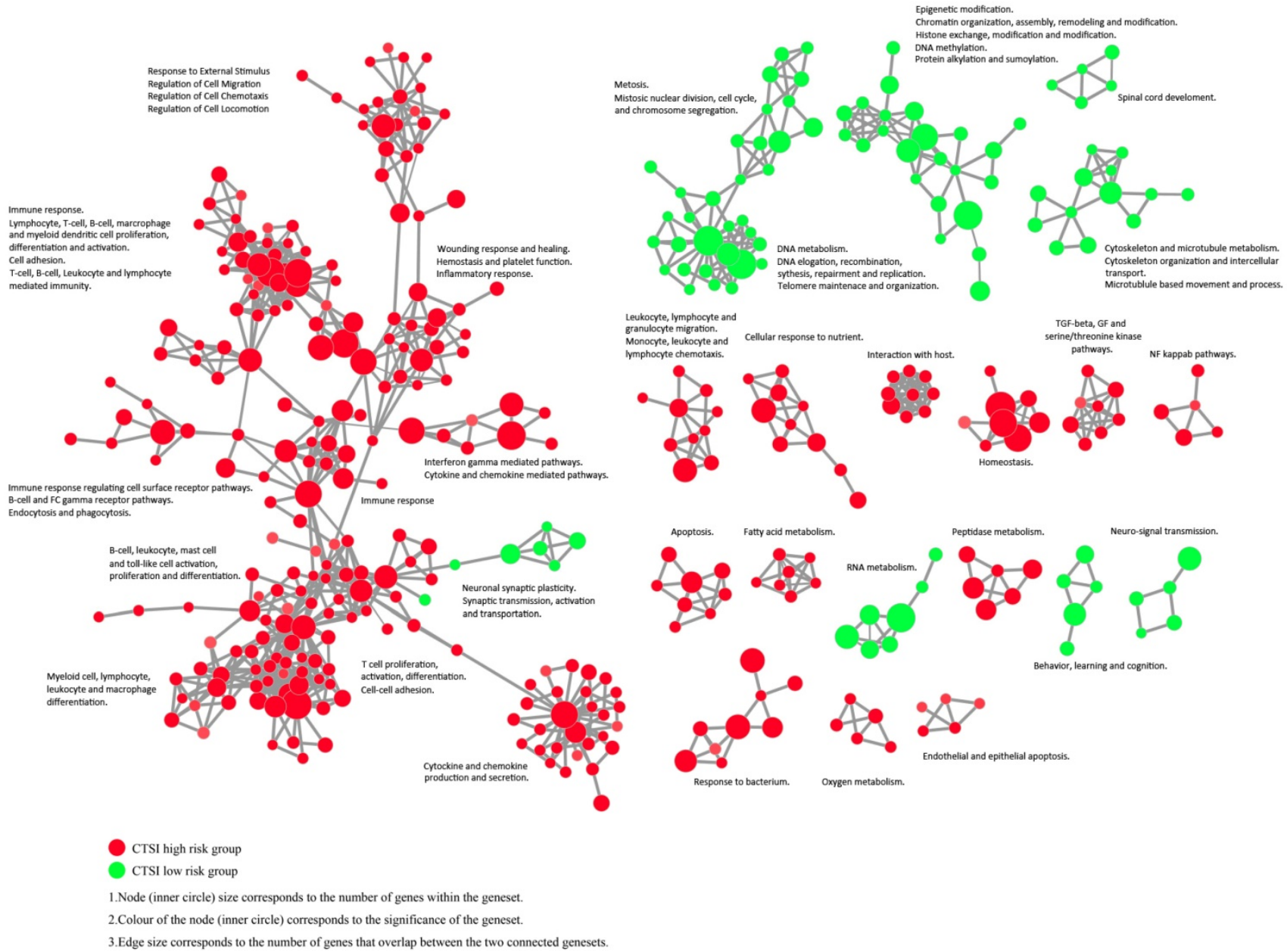

Figure 5. Enrichment map contrasting both CTSI risk score phenotypes. The gene set networks illustrated the results of GSEA targeted GO biological processes contrasting CTSI high-risk and CTSI low-risk phenotypes. Each node represents a gene set. Links between nodes represented the genes shared by both gene sets (filtered with $\mathrm{p}<0.05, \mathrm{FDR}<0.05$, Jaccard coefficient $<0.95$ ). The node color represented the strength and direction of enrichment (red gene sets were enriched in CTSI high-risk phenotype, green ones were enriched in CTSI low-risk phenotype). The figure was made by the Enrichment Map from Cytoscape 3.2.

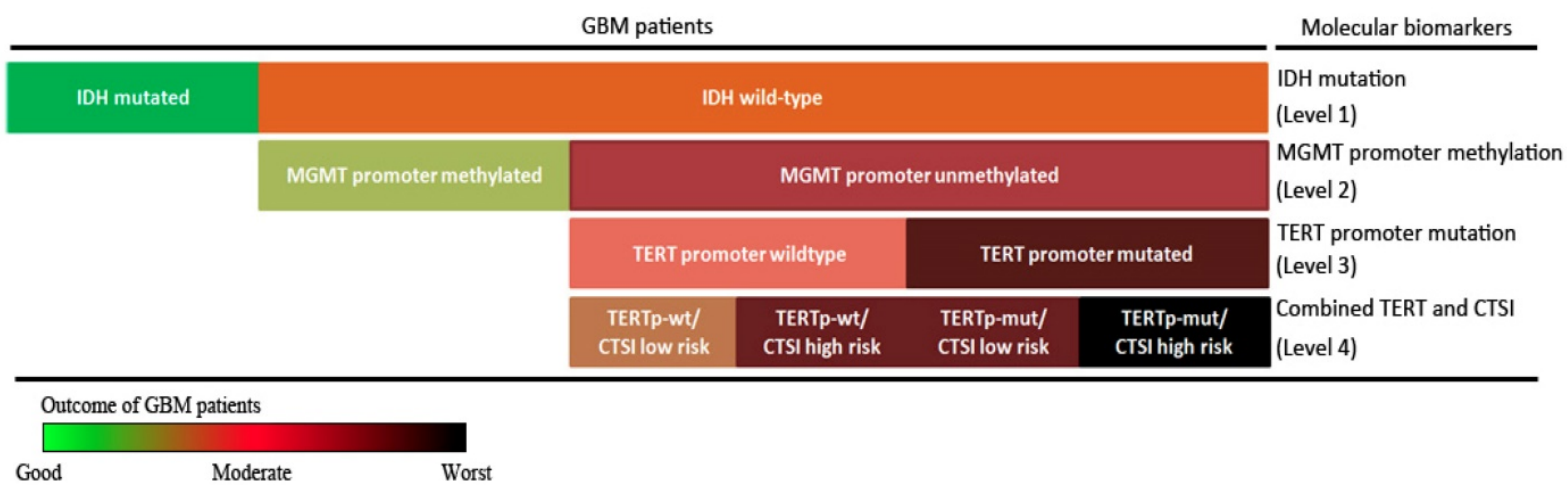

Figure 6. Stepwise strategy for GBM prognosis evaluation. The stepwise GBM prognosis evaluation system combined IDH mutation, MGMT promoter methylation, TERT promoter mutation and CTSI together. The color changed from green to black representing the patients' outcome from the good to the worst. 
prognostic difference of CTSI risk score for overall GBM patients was majorly contributed by IDH-wt and MGMTp-unmeth patients. On the other hand, for GBM patients, TERT promoter mutation was predictive only in IDH-wt/MGMTp-unmeth subgroup. This finding agreed with another study focusing on TERT promoter mutation and MGMT promoter methylation for GBM patients [23]. It suggests that the prognostic value of TERT promoter mutation is also majorly contributed by IDH-wt and MGMTp-unmeth patients. CTSI was a significant prognostic factor for patients with TERT promoter mutation. Based on these results, we suggest CTSI is a significant prognostic indicator for IDH-wt/MGMTp-unmeth GBM patients. And combined with TERT promoter mutation, CTSI is capable of further predicting the prognosis of GBM patients.

Finally, combining IDH mutation, TERT promoter mutation, MGMT promoter methylation with CTSI, we established a stepwise 4-level strategy for GBM prognosis evaluation (Figure 6). First, IDH mutation is the most important factor to divide GBM patients into those with good outcomes (IDH-mut) and bad outcomes (IDH-wt) (level 1). Second, for IDH-wt patients, MGMTp-meth patients have a better prognosis than MGMTp-unmeth patients (level 2). Third, TERT promoter mutation can predict outcome in MGMTp-unmeth patients, but not in MGMTp-meth patients (level 3). Finally, for IDH-wt/MGMTp-unmeth patients, CTSI is an important predictive factor and together with TERT promoter mutation, the prognosis of GBM patients can be more precisely predicted (level 4).

In conclusion, CTSI risk score is a predictable factor for IDH-wt/MGMTp-unmeth GBM patients. Based on CTSI, IDH mutation, TERT promoter mutation and MGMT promoter methylation, a 4-level stepwise prognosis evaluation system providing more precise outcome prediction for GBM patients was established.

\section{Materials and Methods}

\section{Gene expression datasets and clinical data}

GBM gene expression profiles and corresponding clinical data were obtained from three public databases: the TCGA dataset (http://cancergenome.nih.gov), the GEO GSE4271 dataset (https://www.ncbi.nlm.nih.gov/geo/query/ acc.cgi?acc $=$ GSE4271) and the CGGA dataset (http://cgga.org.cn). All these datasets were generated on Affymetrix platform HG-U133a.

Based on TCGA dataset, we selected 195 patients received post-operationally combined radio-chemo- therapy to regain a new cohort, TCGA 195 patient cohort, as training set to identify the gene expression signature. This dataset is a subset of TCGA dataset, and the data was also generated on Affymetrix platform HG-U133a. On the other hand, the TCGA 529 patient cohort, GSE4271 dataset (54 patients) and CGGA dataset (144 patients) were included as validation sets.

\section{Microarray data processing}

The Robust Multichip Average (RMA) algorithm was used for background adjustment [37]. The probes (or probe sets) from Affymetrix HG-U133a were re-mapped to the human genome (GRCh38) using hgu133a.db (R/Bioconductor package). Multiple probes (or probe sets) mapping to the same gene were averaged using the mean values of those probes (or probe sets) to generate a single expression value (on the log2 scale). Two differential gene expression profiles from the TCGA dataset were created. Profile $A$ was the differential gene expression profile between GBM and normal brain tissues. Profile B was generated as the following: 1 . We used the median survival time as the cutoff point, and divided the 195 patient cohort into the radio-chemotherapy resistant group ( $\mathrm{n}=85$, median: 44.6 months) and the radio-chemotherapy sensitive group $(n=110$, median: 79.8 months). 2. By comparing gene expression patterns between the two groups, a differential gene expression profile was generated. As a result, Profile A contained 1349 genes and Profile B contained 1399 genes. Finally, we intersected Profile A with Profile B and a new data matrix containing 137 genes and 195 samples emerged. These 137 genes were GBM tissue-specific and associated with radio-chemotherapy sensitivity. The workflow of data processing was shown in Supplementary Figure S1.

\section{Risk scoring model}

We performed univariable Cox regression analysis to evaluate the relationship between the expression levels of each of the 137 genes and the patient's overall survival (OS) time in the training dataset. Multivariable Cox regression analysis was performed on those genes in the training dataset, with OS as the dependent variable and other clinical information as the covariables. The genes significantly associated with survival $(\mathrm{p}<0.01)$ were used to create a risk scoring model for prognosis prediction. The risk scoring model, which we named Combined Therapy Sensitivity Index, CTSI, was defined as a linear combination of the expression values of the prognostic genes and the multivariable Cox regression coefficients as the weight. The risk score was calculated as previously described [24, 25, 38-41]: 


$$
\mathrm{CTSI}=\sum_{i=1}^{N}\left(\operatorname{Exp}_{i} * \operatorname{Coe}_{i}\right) .
$$

In this formula, $\mathrm{N}$ stands for the number of prognostic genes. Exp $p_{i}$ is the relative expression level of gene ${ }_{i}$ Coe $_{i}$ is the estimated regression coefficient of gene $_{i}$ in the multivariable Cox regression analysis.

\section{Tissue samples, patients' information and follow-up}

To further validate the CTSI risk scoring system, we collected 178 GBM surgical specimens from the tissue archive in our hospital. Those patients were admitted between Oct. 2008 - Nov. 2015. Age-matched normal brain tissues were obtained from patients with severe brain trauma. The expression of those 13 signature genes were quantified by real-time RT-PCR. Post-therapy MR images of the brain was performed to evaluate the response to the recurrence of tumors at 1-year intervals for follow-up. Data on death were defined as failure events for the overall survival rate. All follow-ups in this study ended in May 2016. The study was approved by the Committee on Medicine Ethics of Xiangya Hospital of Central South University. All patients were well informed and written informed consent was provided.

\section{RT-PCR and Quantitative real-time PCR}

The RT-PCR and quantitative real-time PCR were performed as previously described [42]. Briefly, total RNA isolation (Invitrogen), first strand cDNA synthesis (Fermentas) and qPCR (SYBR-Premix Ex TaqTM kit; Takara) were performed according to manufacturer's protocol. Thirty-five cycles were conducted on the ABI PRISM_ 7900 HT. Reported values were calculated using the $2-\Delta \Delta \mathrm{Ct}$ method, normalized against endogenous GAPDH. Quantitative real-time PCR was conducted in triplicate for each sample. For data analysis, gene expression in normal brain tissue was used as reference to calculate relative expression level. Primer sequences were shown in Supplementary Table S1.

\section{IDH mutation, TERT promoter mutation and MGMT promoter methylation}

Genomic DNA from 178 GBM tissue samples was isolated by using the QIAamp DNA mini kit (Qiagen) according to the manufacturer's instructions.

IDH1 and IDH2 mutation analysis were performed using previously described methods by pyrosequencing $[43,44]$. The regions spanning wild-type R132 of IDH1 and wild-type R172 of IDH2 were amplified by PCR. The PCR analysis was performed in $25 \mathrm{ul}$ reaction volume, containing 0.2 $\mathrm{uM}$ each forward and reverse primer, $1 \times$ buffer, 2.0 mM dNTPs, $0.04 \mathrm{U}$ of HotstartTaq (Qiagen), $1 \mathrm{mM}$
$\mathrm{MgCl}_{2}$ and $2 \mathrm{ml}$ of $10-20 \mathrm{ng}$ DNA. The PCR conditions were as follows: $95^{\circ} \mathrm{C}$ for 9 minutes; 45 cycles of $95^{\circ} \mathrm{C}$ for 15 seconds, $56^{\circ} \mathrm{C}$ for 25 seconds, $72^{\circ} \mathrm{C}$ for 1 minute (ABI PCR system 9700). DNA was purified and subjected to pyrosequencing on PyroMark Q96 ID System (QIAGEN). IDH mutated means that the subject was IDH1 or IDH2 mutated. All primers for IDH1 and IDH2 mutation analysis are listed in Supplementary Table S1b.

We used Sanger sequencing to determine the TERT promoter mutational status as described by Eckel-Passow JE and Lachance DH [14]. Sequences covering C228T and C250T mutations in TERT promoter was amplified, which yield a 244-bp product. A total volume of $20 \mathrm{ul}$ reaction mixture was prepared for PCR, consisting of 10-100ng DNA in solution, Platinum Taq DNA polymerase (1.5 unit), $0.2 \mathrm{mM}$ dNTPs, $0.25 \mathrm{mM}$ for each primer and $0.5 \mathrm{X}$ PCR Enhancer. The first PCR cycling conditions were set at $95^{\circ} \mathrm{C}$ for 2 minutes, 40 cycles at $95^{\circ} \mathrm{C}$ for 15 seconds, $62^{\circ} \mathrm{C}$ for 20 seconds and $72{ }^{\circ} \mathrm{C}$ for 10 minutes. $1 \mathrm{uL}$ of the amplified DNA from the first PCR was used for second PCR in a $20 \mathrm{uL}$ reaction mixture, containing $0.5 \times$ PCR Enhancer, $10 \times \mathrm{dNTP}$ mix that contained 1.5 $\mathrm{mM}$ dGTP, 0.5mM deaza-GTP (Sigma-Aldrich) and $0.5 \mathrm{mM}$ primers. The reaction cycling condition of was the same with the first PCR. Products of second PCR were purified and subjected to Sanger sequencing by using the BigDye Terminator cycle sequencing kit (Applied Biosystems) with the forward PCR primer as a sequencing primer. All primers are listed in Supplementary Table S1c.

We analyzed the methylation status of MGMT by bisulfite modification of the genomic DNA followed by pyrosequencing as previously described [45]. We averaged the methylation values across the $16 \mathrm{CpG}$ sites tested within the MGMT promoter. With an average methlyation above $10 \%$, the samples were considered as MGMTp-meth. Primers for MGMT promoter methylation analysis are listed in Supplementary Table S1d.

\section{Gene set enrichment analysis}

Gene Set Enrichment Analysis (GSEA) was used to identify associated signaling pathways between CTSI high-risk and CTSI low-risk groups. 6466 Annotated gene sets were downloaded from the molecular signatures database (MSigDB) C5 GO gene sets collection [46]. Changes in gene expression and gene sets were evaluated using Permutation testing (1,000 permutations). The gene sets with a false discovery rate (FDR) $<0.05$ were considered to be significantly enriched [46]. The GSEA results were visualized using Cytoscape 3.2 and Enrichment Maps $[47,48]$. 


\section{Statistical analysis}

The receiver operating characteristic (ROC) curve was used to evaluate the prognostic performance of CTSI. Youden's index was calculated in the training dataset to identify the cutoff point of the CTSI score. The calculation formula of Youden's J statistic is shown as below:

$$
\mathrm{J}=\text { sensitivity }+ \text { specificity }-1
$$

With the two right-hand quantities being sensitivity and specificity, the formula is:

$$
\begin{aligned}
& \mathrm{J}=\frac{\text { true positives }}{\text { ture positives }+ \text { flase negatives }} \\
& \qquad+\frac{\text { true negatives }}{\text { true negatives }+ \text { false positives }}-1
\end{aligned}
$$

The GBM patients in the training and validation datasets were divided into the high-risk group and the low-risk group. The Kaplan-Meier method was used to estimate survival time for all the datasets and Xiangya GBM cohort. Multivariate Cox regression analysis was performed to test whether CTSI risk score was a significant predictor of survival after taking into account other important variables. The receiver operating characteristic curves was used to analyze the sensitivity and specificity of CTSI risk score in the TCGA 529 patient cohort dataset. Area under the curve (AUC) was calculated based on ROC. All analyses were performed using R/Bio-Conductor (version 3.2.2).

\section{Abbreviations}

GBM: Glioblastoma; TCGA: The Cancer Genome Atlas; GEO: Gene Expression Omnibus; CGGA: Chinese Glioma Genome Atlas; GSEA: Gene set enrichment analysis; GO: Gene Ontology; CTSI: Combined Therapy Sensitivity Index; HR: Hazard Ratio; IDH-wt: IDH wild-type; IDH-mut: IDH-mutant; MGMTp-unmeth: MGMT promoter unmethylated; MGMTp-meth: MGMT promoter methylated; TERTp-mut: TERT promoter mutant; TERTp-wt: TERT promoter wild-type; G-CIMP: Glioma CpG island methylator phenotype; ROC: Receiver operating characteristic; AUC: Area under the curve; OS: Overall survival; RMA: Robust Multichip Average; MSigDB: Molecular signatures database; FDR: False discovery rate.

\section{Supplementary Material}

Supplementary figures and tables. http://www.jcancer.org/v10p5536s1.pdf

\section{Acknowledgements}

This work was supported by the National Natural Science Foundation of China (Grant No. 81472594, 81560414, 81770781).

\section{Author Contributions}

Xuejun Li and Siyi Wanggou conceived and designed the study. Ningrong Ye, Nian Jiang and Chunhai Huang organized revision. Ningrong Ye performed micro array data analysis. Chengyuan Feng, Feiyifan Wang and Yandong Su performed validation experiments. Nian Jiang and Hanwen Zhang finished follow-up and clinical data collection. Harrusin Xiao Bai performed statistical analysis. Li Yang and Chunhai Huang reviewed and edited the manuscript. All the authors have read and approved the manuscript.

\section{Competing Interests}

The authors have declared that no competing interest exists.

\section{References}

1. Sturm D, Bender S, Jones DT, Lichter P, Grill J, Becher O, Hawkins C, Majewski J, Jones C, Costello JF, Iavarone A, Aldape K, Brennan CW, Jabado N and Pfister SM. Paediatric and adult glioblastoma: multiform (epi)genomic culprits emerge. Nature reviews Cancer. 2014; 14(2):92-107.

2. Ostrom QT, Gittleman H, Fulop J, Liu M, Blanda R, Kromer C, Wolinsky Y, Kruchko C and Barnholtz-Sloan JS. CBTRUS Statistical Report: Primary Brain and Central Nervous System Tumors Diagnosed in the United States in 2008-2012. Neuro-oncology. 2015; 17 Suppl 4:iv1-iv62.

3. Van Meir EG, Hadjipanayis CG, Norden AD, Shu HK, Wen PY and Olson JJ. Exciting new advances in neuro-oncology: the avenue to a cure for malignant glioma. CA: a cancer journal for clinicians. 2010; 60(3):166-193.

4. Tanaka S, Louis DN, Curry WT, Batchelor TT and Dietrich J. Diagnostic and therapeutic avenues for glioblastoma: no longer a dead end? Nature reviews Clinical oncology. 2013; 10(1):14-26.

5. Aldape K, Zadeh G, Mansouri S, Reifenberger G and von Deimling A. Glioblastoma: pathology, molecular mechanisms and markers. Acta neuropathologica. 2015; 129(6):829-848.

6. Li QJ, Cai JQ and Liu CY. Evolving Molecular Genetics of Glioblastoma. Chinese medical journal. 2016; 129(4):464-471.

7. Cancer Genome Atlas Research N. Comprehensive genomic characterization defines human glioblastoma genes and core pathways. Nature. 2008; 455(7216):1061-1068.

8. Eder K and Kalman B. Molecular heterogeneity of glioblastoma and its clinical relevance. Pathology oncology research : POR. 2014; 20(4):777-787.

9. Yan $\mathrm{H}$, Parsons DW, Jin G, McLendon R, Rasheed BA, Yuan W, Kos I, Batinic-Haberle I, Jones S, Riggins GJ, Friedman H, Friedman A, Reardon D, Herndon J, Kinzler KW, Velculescu VE, et al. IDH1 and IDH2 mutations in gliomas. The New England journal of medicine. 2009; 360(8):765-773.

10. Wen PY and Kesari S. Malignant gliomas in adults. The New England journal of medicine. 2008; 359(5):492-507.

11. Parsons DW, Jones S, Zhang X, Lin JC, Leary RJ, Angenendt P, Mankoo P, Carter H, Siu IM, Gallia GL, Olivi A, McLendon R, Rasheed BA, Keir S, Nikolskaya T, Nikolsky $\mathrm{Y}$, et al. An integrated genomic analysis of human glioblastoma multiforme. Science. 2008; 321(5897):1807-1812.

12. Brennan CW, Verhaak RG, McKenna A, Campos B, Noushmehr H, Salama SR, Zheng S, Chakravarty D, Sanborn JZ, Berman SH, Beroukhim R, Bernard B, Wu CJ, Genovese G, Shmulevich I, Barnholtz-Sloan J, et al. The somatic genomic landscape of glioblastoma. Cell. 2013; 155(2):462-477.

13. Mosrati MA, Malmstrom A, Lysiak M, Krysztofiak A, Hallbeck M, Milos P, Hallbeck AL, Bratthall C, Strandeus M, Stenmark-Askmalm M and Soderkvist $P$. TERT promoter mutations and polymorphisms as prognostic factors in primary glioblastoma. Oncotarget. 2015; 6(18):16663-16673.

14. Eckel-Passow JE, Lachance DH, Molinaro AM, Walsh KM, Decker PA, Sicotte H, Pekmezci M, Rice T, Kosel ML, Smirnov IV, Sarkar G, Caron AA, Kollmeyer TM, Praska CE, Chada AR, Halder C, et al. Glioma Groups Based on $1 p / 19 q$, IDH, and TERT Promoter Mutations in Tumors. The New England journal of medicine. 2015; 372(26):2499-2508

15. Hegi ME, Diserens AC, Gorlia T, Hamou MF, de Tribolet N, Weller M, Kros JM, Hainfellner JA, Mason W, Mariani L, Bromberg JE, Hau P, Mirimanoff RO, Cairncross JG, Janzer RC and Stupp R. MGMT gene silencing and benefit from temozolomide in glioblastoma. The New England journal of medicine. 2005; 352(10):997-1003.

16. Verhaak RG, Hoadley KA, Purdom E, Wang V, Oi Y, Wilkerson MD, Miller CR, Ding L, Golub T, Mesirov JP, Alexe G, Lawrence M, O'Kelly M, Tamayo P, Weir BA, Gabriel S, et al. Integrated genomic analysis identifies clinically relevant subtypes of glioblastoma characterized by abnormalities in PDGFRA, IDH1, EGFR, and NF1. Cancer cell. 2010; 17(1):98-110. 
17. Noushmehr $\mathrm{H}$, Weisenberger DJ, Diefes $\mathrm{K}$, Phillips HS, Pujara $\mathrm{K}$, Berman BP, Pan F, Pelloski CE, Sulman EP, Bhat KP, Verhaak RG, Hoadley KA, Hayes DN, Perou CM, Schmidt HK, Ding L, et al. Identification of a $\mathrm{CpG}$ island methylator phenotype that defines a distinct subgroup of glioma. Cancer cell. 2010; 17(5):510-522.

18. Louis DN, Perry A, Reifenberger G, von Deimling A, Figarella-Branger D, Cavenee WK, Ohgaki H, Wiestler OD, Kleihues P and Ellison DW. The 2016 World Health Organization Classification of Tumors of the Central Nervous System: a summary. Acta neuropathologica. 2016; 131(6):803-820.

19. Bai H, Harmanci AS, Erson-Omay EZ, Li J, Coskun S, Simon M, Krischek B, Ozduman K, Omay SB, Sorensen EA, Turcan S, Bakirciglu M, Carrion-Grant G, Murray PB, Clark VE, Ercan-Sencicek AG, et al. Integrated genomic characterization of IDH1-mutant glioma malignant progression. Nature genetics. 2016; 48(1):59-66.

20. Weller $M$, van den Bent $M$, Hopkins $K$, Tonn JC, Stupp R, Falini A, Cohen-Jonathan-Moyal E, Frappaz D, Henriksson R, Balana C, Chinot O, Ram Z, Reifenberger G, Soffietti R, Wick W and European Association for Neuro-Oncology Task Force on Malignant G. EANO guideline for the diagnosis and treatment of anaplastic gliomas and glioblastoma. The Lancet Oncology. 2014; 15(9):e395-403.

21. Weller M, Pfister SM, Wick W, Hegi ME, Reifenberger G and Stupp R. Molecular neuro-oncology in clinical practice: a new horizon. The Lancet Oncology. 2013; 14(9):e370-379.

22. Stupp R, Brada M, van den Bent MJ, Tonn JC, Pentheroudakis G and Group EGW. High-grade glioma: ESMO Clinical Practice Guidelines for diagnosis, treatment and follow-up. Annals of oncology : official journal of the European Society for Medical Oncology / ESMO. 2014; 25 Suppl 3:iii93-101.

23. Arita $\mathrm{H}$, Yamasaki $\mathrm{K}$, Matsushita $\mathrm{Y}$, Nakamura $\mathrm{T}$, Shimokawa A, Takami $\mathrm{H}$, Tanaka S, Mukasa A, Shirahata M, Shimizu S, Suzuki K, Saito K, Kobayashi K, Higuchi F, Uzuka T, Otani R, et al. A combination of TERT promoter mutation and MGMT methylation status predicts clinically relevant subgroups of newly diagnosed glioblastomas. Acta neuropathologica communications. 2016; 4(1):79.

24. Zhang CB, Zhu P, Yang P, Cai JQ, Wang ZL, Li QB, Bao ZS, Zhang $W$ and Jiang $\mathrm{T}$. Identification of high risk anaplastic gliomas by a diagnostic and prognostic signature derived from mRNA expression profiling. Oncotarget. 2015; 6(34):36643-36651.

25. Wang W, Zhang L, Wang Z, Yang F, Wang H, Liang T, Wu F, Lan Q, Wang J and Zhao J. A three-gene signature for prognosis in patients with MGMT promoter-methylated glioblastoma. Oncotarget. 2016.

26. Zhong J, Bach CT, Shum MS and O'Neill GM. NEDD9 regulates 3D migratory activity independent of the Rac1 morphology switch in glioma and neuroblastoma. Molecular cancer research : MCR. 2014; 12(2):264-273.

27. Geng P, Ou J, Li J, Liao Y, Wang N, Xie G, Sa R, Liu C, Xiang L and Liang H. A Comprehensive Analysis of Influence ERCC Polymorphisms Confer on the Development of Brain Tumors. Molecular neurobiology. 2016; 53(4):2705-2714.

28. Ren M, Zhou C, Liang H, Wang X and Xu L. RNAi-Mediated Silencing of EIF3D Alleviates Proliferation and Migration of Glioma U251 and U87MG Cells. Chemical biology \& drug design. 2015; 86(4):715-722.

29. Yang LS, Xu XE, Liu XP, Jin H, Chen ZQ, Liu XH, Wang Y, Huang FP and Shi Q. iTRAQ-based quantitative proteomic analysis for identification of oligodendroglioma biomarkers related with loss of heterozygosity on chromosomal arm 1p. Journal of proteomics. 2012; 77:480-491.

30. Szulzewsky F, Pelz A, Feng X, Synowitz M, Markovic D, Langmann T, Holtman IR, Wang X, Eggen BJ, Boddeke HW, Hambardzumyan D, Wolf SA and Kettenmann H. Glioma-associated microglia/macrophages display an expression profile different from M1 and M2 polarization and highly express Gpnmb and Spp1. PloS one. 2015; 10(2):e0116644.

31. Wang Y, Wei Y, Tong H, Chen L, Fan Y, Ji Y, Jia W, Liu D and Wang G. MiR-302c-3p suppresses invasion and proliferation of glioma cells via down-regulating metadherin $(\mathrm{MTDH})$ expression. Cancer biology \& therapy. 2015; 16(9):1308-1315.

32. Qu DW, Liu Y, Wang L, Xiong Y, Zhang CL and Gao DS. Glial cell line-derived neurotrophic factor promotes proliferation of neuroglioma cells by up-regulation of cyclins PCNA and Ki-67. European review for medical and pharmacological sciences. 2015; 19(11):2070-2075.

33. Oram SW, Ai J, Pagani GM, Hitchens MR, Stern JA, Eggener S, Pins M, Xiao W, Cai X, Haleem R, Jiang F, Pochapsky TC, Hedstrom L and Wang Z. Expression and function of the human androgen-responsive gene ADI1 in prostate cancer. Neoplasia. 2007; 9(8):643-651.

34. Zhu XS, Dai YC, Chen ZX, Xie JP, Zeng W, Lin YY and Tan QH. Knockdown of ECHS1 protein expression inhibits hepatocellular carcinoma cell proliferation via suppression of Akt activity. Critical reviews in eukaryotic gene expression. 2013; 23(3):275-282.

35. Xie X, Li F, Wang Y, Wang Y, Lin Z, Cheng X, Liu J, Chen C and Pan L. Molecular basis of ubiquitin recognition by the autophagy receptor CALCOCO2. Autophagy. 2015; 11(10):1775-1789.

36. Stupp R, Hegi ME, Mason WP, van den Bent MJ, Taphoorn MJ, Janzer RC, Ludwin SK, Allgeier A, Fisher B, Belanger K, Hau P, Brandes AA, Gijtenbeek J, Marosi C, Vecht CJ, Mokhtari K, et al. Effects of radiotherapy with concomitant and adjuvant temozolomide versus radiotherapy alone on survival in glioblastoma in a randomised phase III study: 5-year analysis of the EORTC-NCIC trial. The Lancet Oncology. 2009; 10(5):459-466.
37. Irizarry RA, Hobbs B, Collin F, Beazer-Barclay $\mathrm{YD}$, Antonellis KJ, Scherf U and Speed TP. Exploration, normalization, and summaries of high density oligonucleotide array probe level data. Biostatistics. 2003; 4(2):249-264.

38. Zhou M, Guo M, He D, Wang X, Cui Y, Yang H, Hao D and Sun J. A potential signature of eight long non-coding RNAs predicts survival in patients with non-small cell lung cancer. Journal of translational medicine. 2015; 13:231.

39. Meng J, Li P, Zhang $\mathrm{Q}$, Yang Z and Fu S. A four-long non-coding RNA signature in predicting breast cancer survival. Journal of experimental \& clinical cancer research : CR. 2014; 33:84

40. Cheng W, Ren X, Cai J, Zhang C, Li M, Wang K, Liu Y, Han S and Wu A. A five-miRNA signature with prognostic and predictive value for MGMT promoter-methylated glioblastoma patients. Oncotarget. 2015; 6(30):29285-29295.

41. Bao ZS, Li MY, Wang JY, Zhang CB, Wang HJ, Yan W, Liu YW, Zhang W, Chen $\mathrm{L}$ and Jiang T. Prognostic value of a nine-gene signature in glioma patients based on mRNA expression profiling. CNS neuroscience \& therapeutics. 2014; 20(2):112-118.

42. Wanggou S, Jiang X, Li Q, Zhang L, Liu D, Li G, Feng X, Liu W, Zhu B, Huang $W$, Shi J, Yuan $X$ and Ren C. HESRG: a novel biomarker for intracranial germinoma and embryonal carcinoma. Journal of neuro-oncology. 2012; 106(2):251-259.

43. Yan W, Zhang W, You G, Bao Z, Wang Y, Liu Y, Kang C, You Y, Wang L and Jiang T. Correlation of IDH1 mutation with clinicopathologic factors and prognosis in primary glioblastoma: a report of 118 patients from China. PloS one. 2012; 7(1):e30339.

44. Christensen BC, Smith AA, Zheng S, Koestler DC, Houseman EA, Marsit CJ, Wiemels JL, Nelson HH, Karagas MR, Wrensch MR, Kelsey KT and Wiencke JK. DNA methylation, isocitrate dehydrogenase mutation, and survival in glioma. Journal of the National Cancer Institute. 2011; 103(2):143-153.

45. Mulholland S, Pearson DM, Hamoudi RA, Malley DS, Smith CM, Weaver JM, Jones DT, Kocialkowski S, Backlund LM, Collins VP and Ichimura K. MGMT CpG island is invariably methylated in adult astrocytic and oligodendroglial tumors with IDH1 or IDH2 mutations. International journal of cancer. 2012; 131(5):1104-1113.

46. Subramanian A, Tamayo P, Mootha VK, Mukherjee S, Ebert BL, Gillette MA, Paulovich A, Pomeroy SL, Golub TR, Lander ES and Mesirov JP. Gene set enrichment analysis: a knowledge-based approach for interpreting genome-wide expression profiles. Proceedings of the National Academy of Sciences of the United States of America. 2005; 102(43):15545-15550.

47. Shannon P, Markiel A, Ozier O, Baliga NS, Wang JT, Ramage D, Amin N, Schwikowski B and Ideker T. Cytoscape: a software environment for integrated models of biomolecular interaction networks. Genome research. 2003; 13(11):2498-2504

48. Merico D, Isserlin R, Stueker O, Emili A and Bader GD. Enrichment map: a network-based method for gene-set enrichment visualization and interpretation. PloS one. 2010; 5(11):e13984. 